\title{
Imaging science at El Leoncito, Argentina
}

\author{
C. Martinis ${ }^{1}$, J. Baumgardner ${ }^{1}$, S. M. Smith ${ }^{1}$, M. Colerico ${ }^{1, *}$, and M. Mendillo ${ }^{1}$ \\ ${ }^{1}$ Center for Space Physics-Boston, Boston, MA 02215, USA \\ *now at: MIT Haystack Observatory, Westford, MA, USA
}

Received: 1 September 2005 - Revised: 7 February 2006 - Accepted: 22 February 2006 - Published: 3 July 2006

Part of Special Issue "The 11th International Symposium on Equatorial Aeronomy (ISEA-11), Taipei, May 2005"

\begin{abstract}
Thermospheric and mesospheric structures are studied using an all-sky imager located at El Leoncito, Argentina $\left(31.8^{\circ} \mathrm{S}, 69.3^{\circ} \mathrm{W},-18^{\circ} \mathrm{mag}\right.$ lat). This site has relatively high geographic latitude for a location under the crest of the equatorial ionization anomaly (EIA), and thus observations can be used to study the intrusion of several equatorial processes into the midlatitude domain. In addition, it has a conjugate point close to the field of view of our companion imager at Arecibo, PR, allowing for the study of interhemispheric effects. Four types of phenomena were studied using $630.0 \mathrm{~nm}$ and $777.4 \mathrm{~nm}$ observations: (1) highlystructured airglow depletions associated with the RayleighTaylor instability/equatorial spread-F (RTI/ESF) process, (2) brightness waves (BW) associated with the midnight temperature maximum (MTM), (3) strong airglow enhancements associated with the positive phase of ionospheric storms, and (4) simple (non-structured) bands of airglow depletions with characteristics matching a Perkins-like instability. Using $557.7 \mathrm{~nm}$ mesospheric observations, a fifth category of study deals with gravity waves probably generated by lower atmospheric disturbances, and mesospheric bores related to strong vertical temperature gradients.

While ESF depletions and BW events are detected fairly frequently, the mid-latitude bands are not, and thus their successful imaging at El Leoncito offers the first example of the coupling from mid-latitudes to low-latitudes in the South American longitude sector. Preliminary results on these features are presented in this paper. Taken together, these five types of optical structures offer the opportunity to investigate coupling, both in altitude and latitude, of aeronomic processes at low latitudes in an under-sampled longitude sector in the Southern Hemisphere.
\end{abstract}

Keywords. Ionosphere (Equatorial ionosphere; Midlatitude ionosphere; Instruments and techniques)

Correspondence to: C. Martinis

(martinis@bu.edu)

\section{Introduction}

All-sky imager data provide insights into studies of many upper atmospheric phenomena. The roles of optical images are twofold: (1) they offer wide spatial coverage giving the 2-D context for other diagnostic systems that probe the atmosphere along specific lines of sight, e.g., radars, lidars, rockets and satellites; (2) they provide independent science yield of phenomena illuminated by their optical characteristics. One of the advantages of using an all-sky imager is its ability to capture 2-D structures simultaneously within the field-of-view (FOV), in contrast to photometers that cannot capture the full feature simultaneously (they can scan or wait for the structure to move overhead). It is the dramatic spatial signature of a dynamical process that makes imaging science a forefront contributor to aeronomy.

At Boston University, a series of all-sky camera (ASC) were developed, first film-based and then CCD-based, and field campaigns were conducted to exploit this new optical capability for equatorial aeronomy. At Ascension Island in the Atlantic Ocean (Mendillo and Baumgardner, 1982), at Natal, Brazil (Mendillo et al., 1985), at Cachoeira Paulista, Brazil (Sahai et al., 1998), at Kwajalein in the Pacific (Mendillo et al., 1992), at Arequipa, Peru, (Mendillo et al., 1997a) and in Tucumán, Argentina (Martinis et al., 2003), ASC were used to study the signatures of the ESF instability. In such cases, it was the reduction of $630.0 \mathrm{~nm}$ emission (airglow depletions) that captured the ESF's spatial characteristics. Yet, at Arequipa, a second class of $630.0 \mathrm{~nm}$ structure was also detected, termed a brightness wave (BW) by Colerico et al. (1996), a propagating airglow enhancement driven by winds from the midnight temperature maximum (MTM) that occurs near the geographic equator.

A dramatic case of un-anticipated optical structures occurred during the first operation of an ASC for thermospheric-ionospheric studies at midlatitudes at the Arecibo Observatory. Two observing campaigns (in 1993

Published by Copernicus GmbH on behalf of the European Geosciences Union. 


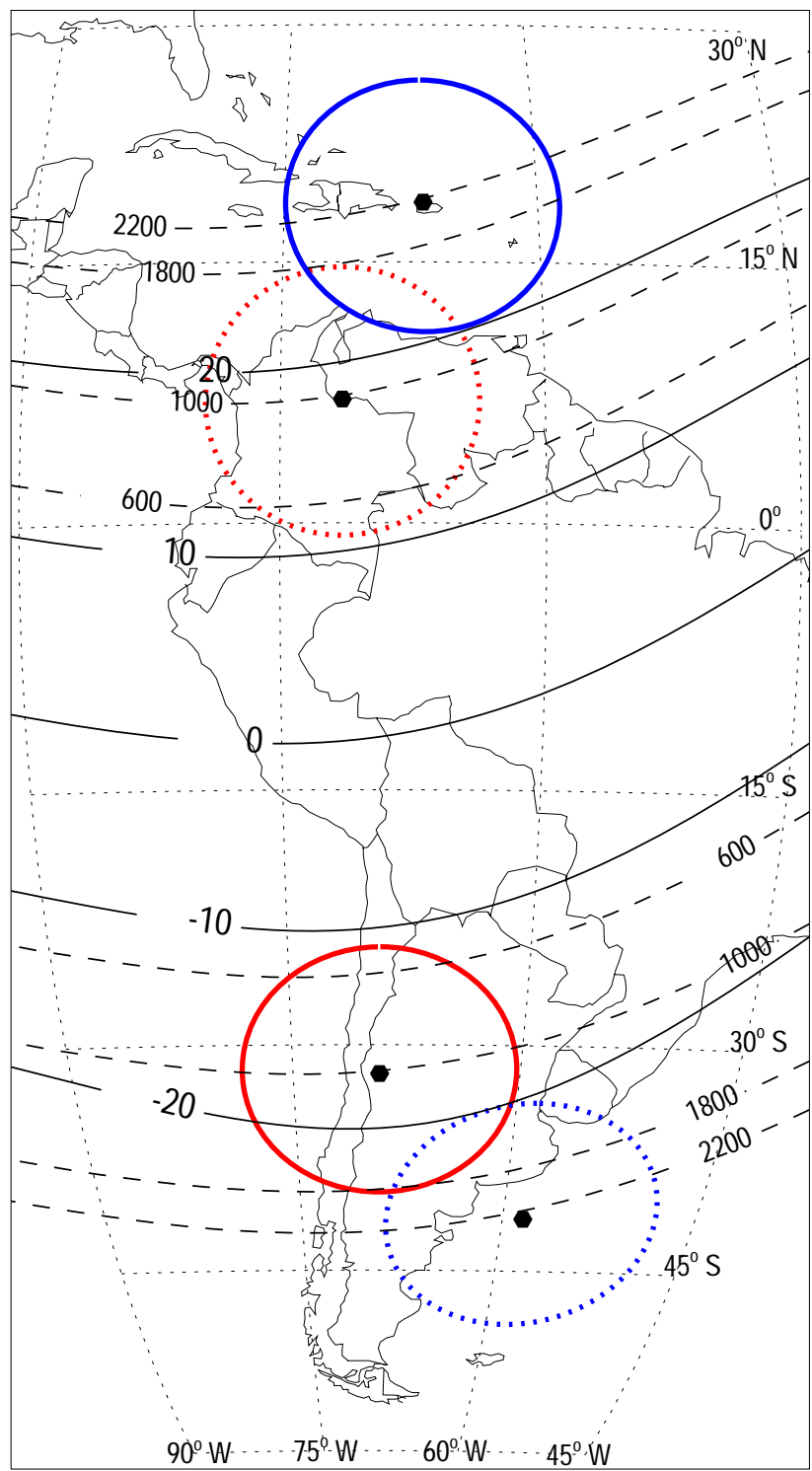

Fig. 1. Map showing the location of the El Leoncito $\left(31.8^{\circ} \mathrm{S}\right)$ and Arecibo $\left(18.3^{\circ} \mathrm{N}\right)$ imagers. Red and blue circles indicate $75^{\circ} \mathrm{FOV}$ for an emission height of $\sim 300 \mathrm{~km}$ for El Leoncito and Arecibo, respectively. Also shown are their respective conjugate FOVs (dotted circles). Apex heights are indicated by dashed lines and magnetic latitudes by solid lines.

and 1995) revealed a robust set of airglow structures (Mendillo et al., 1997b; Miller et al., 1997) that brought new groups into the optical community and generated considerable interest in dynamical processes at the interface between the equatorial aeronomy domain and midlatitudes. The effects observed included a remarkable spectrum of airglow depletions; some were interpreted as signatures of traveling ionospheric disturbances (TIDs) and others as ESF effects found to be at extraordinary distances from the geomagnetic equator (Mendillo et al., 1997b). In follow up work (Miller et al., 1997; Kelley et al., 2002), the TIDs were hypothesized to be a form of the Perkins instability (Perkins, 1973); the possibility of ESF effects reaching Arecibo's location at $\mathrm{L} \sim 1.3$ remained surprising and controversial (Kelley et al., 2000).

An ASC in the Southern Hemisphere (at a longitude comparable to Arecibo's) offers a way to test if processes ordered by geomagnetic fluxtube geometries (such as ESF) were seen in conjugate regions. Moreover, documentation of the Perkins-like instability had not been achieved optically in the American sector in the Southern Hemisphere, and the MTM brightness wave's full latitude extent had also not been measured in the Southern Hemisphere. Finally, optical structures (gravity waves and mesospheric bores) seen in the mesosphere above Arecibo had been sparsely recorded in the Southern Hemisphere using ASC. For all of these reasons, we established a permanent airglow observing station at the Complejo Astronómico El Leoncito $\left(31.8^{\circ} \mathrm{S}, 69.3^{\circ} \mathrm{W}\right.$, $-18^{\circ}$ mag lat), in Argentina. Figure 1 shows why this site was selected as an excellent location to search for effects comparable to those at Arecibo $\left(18.35^{\circ} \mathrm{N}, 66.7^{\circ} \mathrm{W}, 27^{\circ} \mathrm{mag}\right.$ lat). Depicted on this map are the all-sky fields-of-view (FOV) of Arecibo and El Leoncito using blue and red solid line circles, respectively, for a $300 \mathrm{~km}$ emission layer. The blue dotted circle near Arecibo and the red dotted circle near El Leoncito are the conjugate FOVs for El Leoncito and Arecibo, respectively. The solid curved lines give the location of the geomagnetic equator, and $\pm 10^{\circ}$ and $\pm 20^{\circ}$ magnetic latitude lines represent the domain of the equatorial ionization anomaly (EIA) and its optical signature, the inter-tropical arcs. The dashed lines give geomagnetic apex heights, i.e., the altitudes reached at the magnetic equator by the geomagnetic field lines coming from those locations. Thus, El Leoncito at zenith has an apex height of $\sim 1000 \mathrm{~km}$, the typical location of the southern intertropical arc. The northern crest of the EIA is far equatorward at Arecibo where zenith refers to apex heights $>2000 \mathrm{~km}$. Thus, in selecting El Leoncito we are able to track fluxtube-ordered processes over a broad range of latitudes and apex heights. Finally, it is important to mention that optical aeronomy in Brazil has been conducted for decades (Sobral et al., 2002; Sahai et al., 2000), in a longitude section with a considerably different magnetic field declination than at El Leoncito, an important factor in seasonal occurrence patterns for ESF. Thus, longterm optical studies at El Leoncito also provide comparisons with those in a nearby, but significantly different longitude in South America.

In this paper, we report on the comprehensive set of results obtained in the first years of operation at El Leoncito. It is the counterpart to earlier reports of imaging science yields from our sites at Arequipa (Mendillo et al., 1997a) and Arecibo (Mendillo et al., 1997b). It covers several areas of coupling from equatorial to lower midlatitudes, aspects of equatorial aeronomy not yet possible at many other locations. 

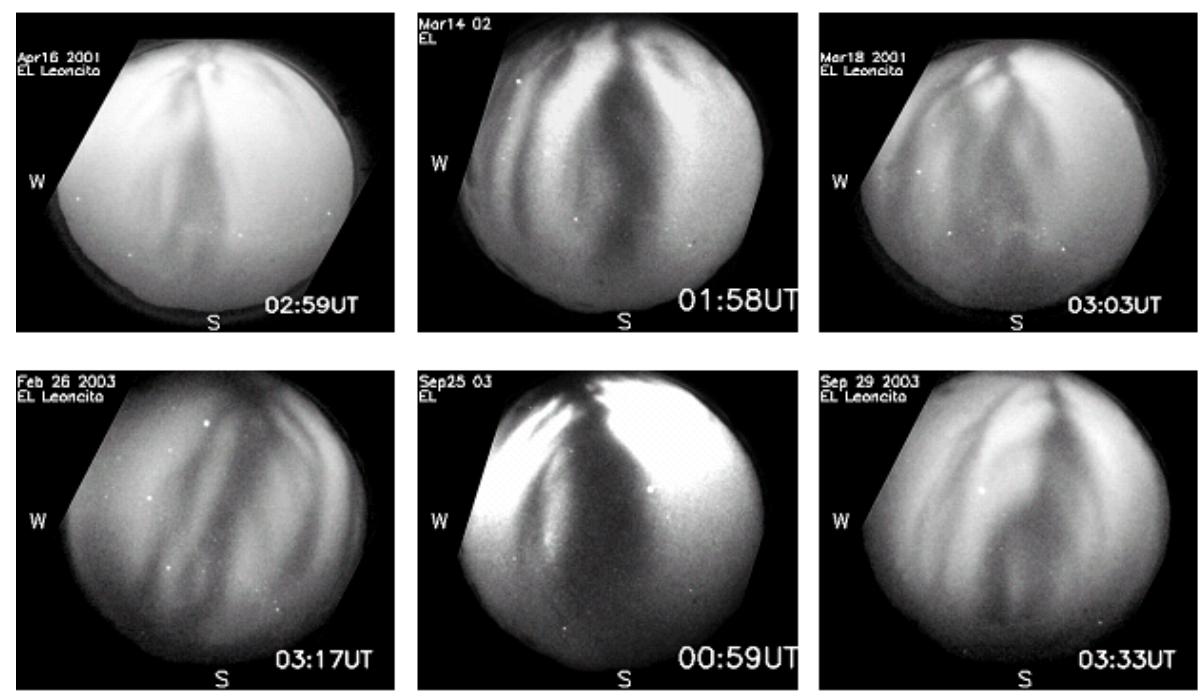

Fig. 2. El Leoncito $630.0 \mathrm{~nm}$ images on six different nights showing typical airglow depletions throughout the night (LT $\approx$ UT-5). North is at the top and west to the left of each image. It is not rare to observe depletions leaving the southern FOV which, as shown in Fig. 1, have apex heights greater than $1800 \mathrm{~km}$.

\section{Instrumentation and data}

In 1999 an all-sky imager was installed by the Boston University Imaging Science Group at the Complejo Astronómico El Leoncito, Argentina $\left(31.8^{\circ} \mathrm{S}, 69.3^{\circ} \mathrm{W}, 18^{\circ} \mathrm{S}\right.$ mag lat). The system utilizes an image-intensified charge-coupled device (ICCD) behind a rotating filter-wheel which houses up to six narrow-band (1.2-1.8 nm FWHM) filters (for further details on the system, see Baumgardner et al. (1993). The filters record several oxygen emissions from different height regimes: mesospheric $\mathrm{O}\left({ }^{1} \mathrm{~S}\right)$ at $557.7 \mathrm{~nm}$ from $\sim 96 \mathrm{~km}$ in altitude, thermospheric $\mathrm{O}\left({ }^{1} \mathrm{D}\right)$ at $630.0 \mathrm{~nm}$ emission from $\sim 250-300 \mathrm{~km}$, and $\mathrm{O}\left({ }^{5} \mathrm{P}\right)$ emission at $777.4 \mathrm{~nm}$ from above $300 \mathrm{~km}$. A filter centered at $644.4 \mathrm{~nm}$ is used to monitor continuum (i.e., non-atmospheric) emission and the two other positions are used for calibration purposes. The system operates for the 14-day moon-down period each month. At this site, clear nights occurred on about an $80 \%$ basis during the years 2000-2005.

Three types of recurrent thermospheric features have been observed using the $630.0 \mathrm{~nm}$ filter: highly-structured airglow depletions that we will associate with ESF events, brightness wave (BW) events driven by the midnight temperature maximum, and "dark bands" that we will associate with the Perkins-like instability, called mid-latitude instabilities (MLI) or mesoscale traveling ionospheric disturbances (MSTID). ESF and BW events occurred $\sim 40 \%$ of the clear nights. But in less than $5 \%$ of the observing nights MLI features were observed. We found the occurrence rate of airglow depletions to have semi-annual peaks during the equinoxes, with an absolute minimum during the June solstice, an annual pattern which is typical of this longitude sector (Aarons,
1993; Tsunoda, 1985). The BW events occur as frequently as ESF events, but no clear seasonal patterns have emerged from the observations. The MLI are more irregular and may be considered as simply episodic in nature.

During large geomagnetic storms we found in two wavelengths $(630.0 \mathrm{~nm}$ and $777.4 \mathrm{~nm})$ strong airglow enhancements caused by a dramatic positive phase ionospheric storm.

Finally, emissions at $557.7 \mathrm{~nm}$ allow us to study mesospheric features associated with lower altitude forcing mechanisms. Specifically, gravity waves, bore events, and tidal variations have been detected with the system.

\section{Thermospheric-ionospheric structures}

\subsection{Airglow depletions associated with ESF}

The association of airglow depletions with equatorial ionospheric irregularities is a well-established fact. The goal of our ESF studies is not to re-demonstrate this, but rather to show that the optical signatures of ESF can reach into the lower-midlatitudes in the Arecibo longitude sector. Due to the flux-tube nature of the ESF process, detection of these irregularities at latitudes with apex heights well above $1400 \mathrm{~km}$ can be used to characterize their intrusion into the midlatitude domains of both hemispheres (Mendillo et al., 2005). Examples of such depletions are shown in Fig. 2. In each of the six cases, most of the depletions extend past zenith, often with complex structuring, indicative of fully-developed ESF plumes of irregularities. El Leoncito has a large number of cases like the ones showed in Fig. 2 with depletions leaving the southern edge of the FOV (mag lat $>20^{\circ} \mathrm{S}$ ). To confirm their ESF characteristic signatures, Fig. 3 gives 

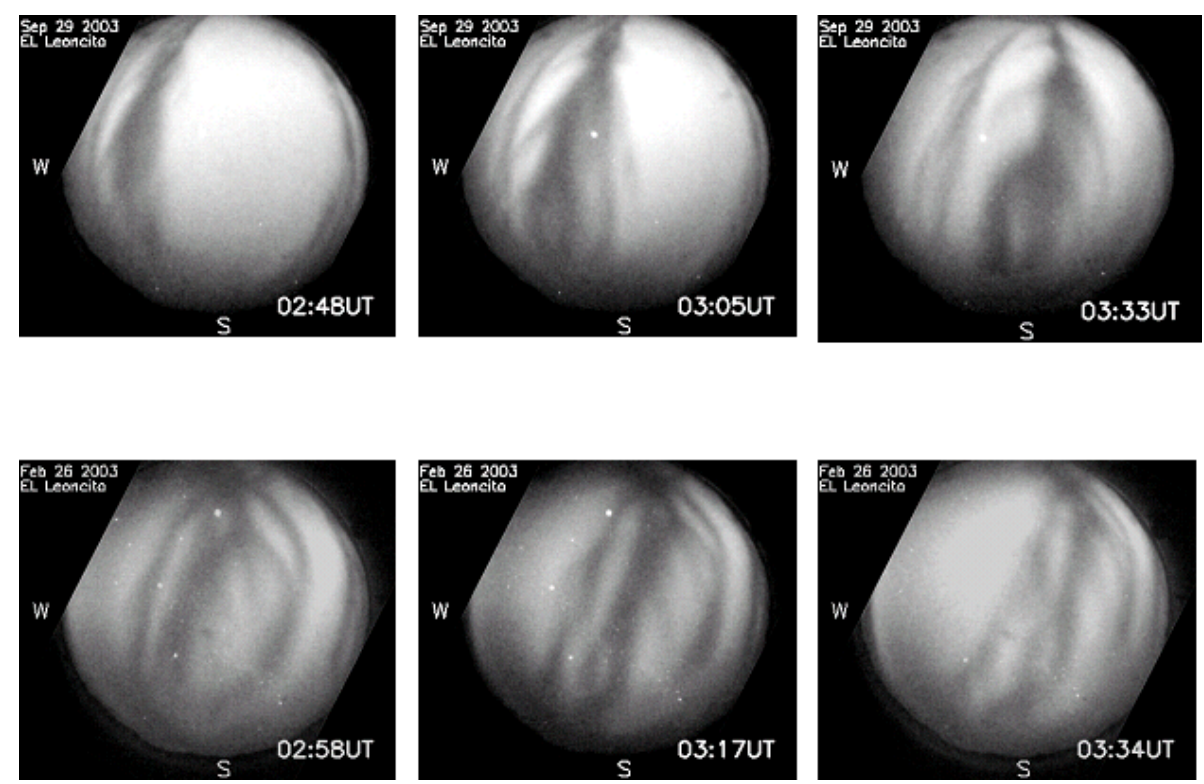

Fig. 3. Examples of eastward moving $630.0 \mathrm{~nm}$ airglow depletions during a 1-h period for two of the cases shown in Fig. 2: 29 September 2003 (top panel); and 26 February 2003 (bottom panel).

unsa

UT Date: February 26,2003
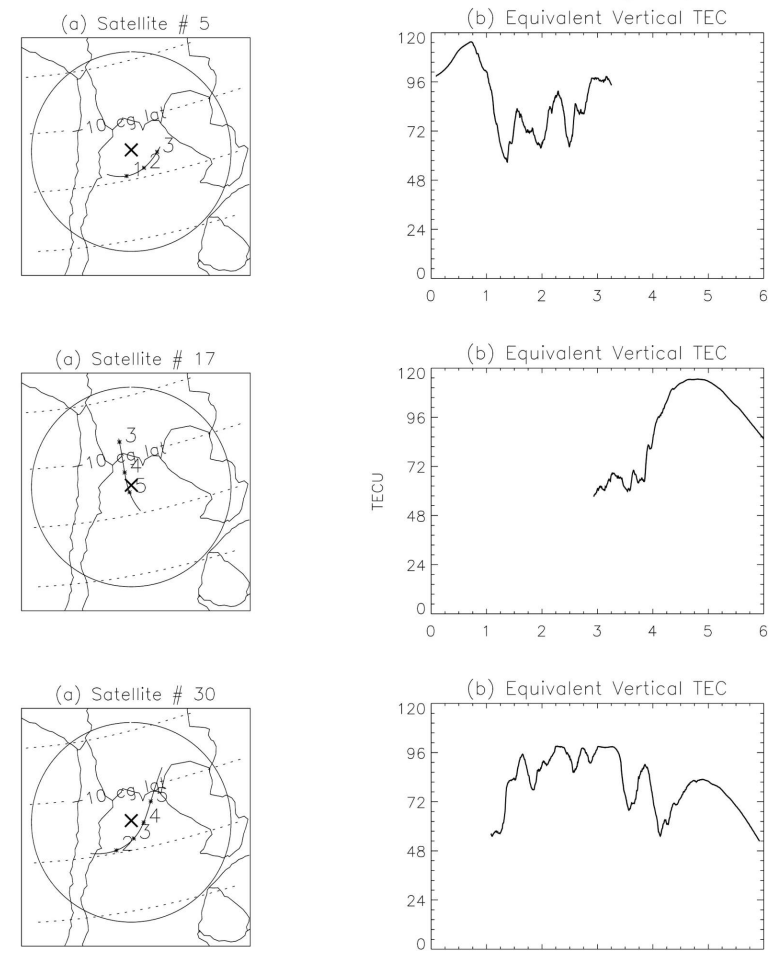
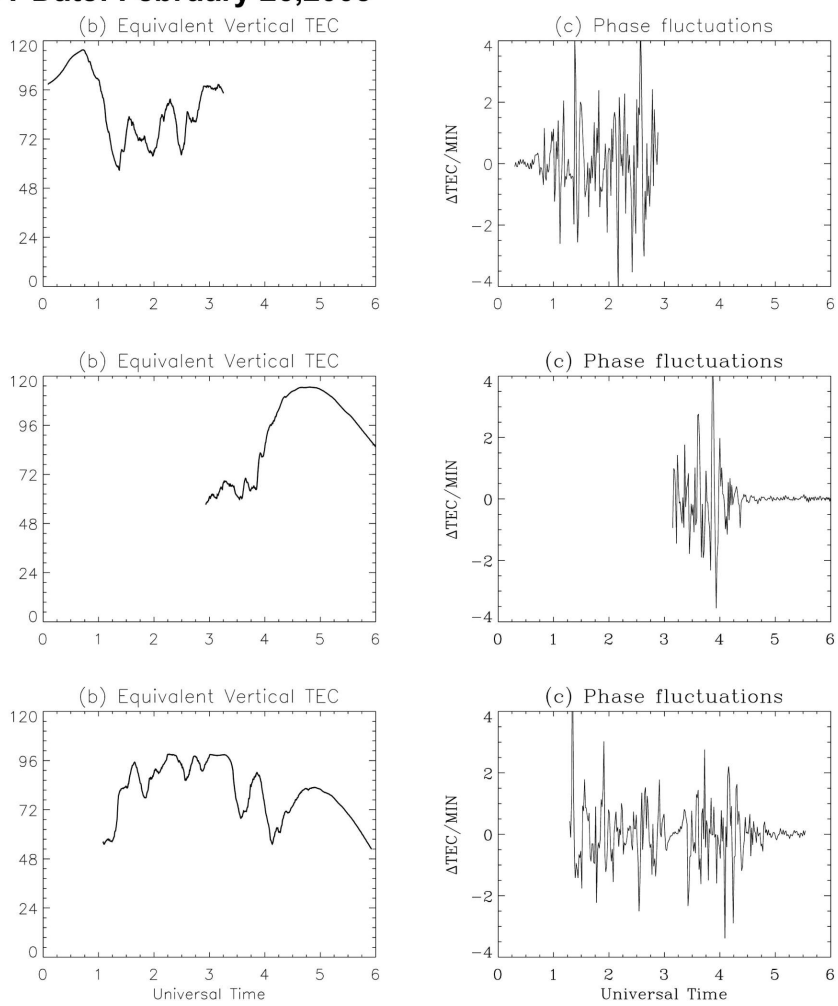

Fig. 4. GPS observations from UNSA $\left(24.73^{\circ} \mathrm{S}, 65.41^{\circ} \mathrm{W}\right)$ for the night of 26 February 2003 , coincident with the imaging data shown in Fig. 3 (bottom panel). (a) the passes of three satellites are shown. The circles are at $350 \mathrm{~km}$ and $15^{\circ}$ elevation angle; (b) vertical total electron content (TEC), in units of $10^{16} \mathrm{el} / \mathrm{m}^{2}$, plotted as a function of universal time, showing the presence of TEC depletions; (c) corresponding phase fluctuations (or total electron content rate of change, dTEC/dt). The temporal/spatial coherence of airglow depletions, TEC reductions, and enhanced phase fluctuations are standard signatures of ESF patterns. 


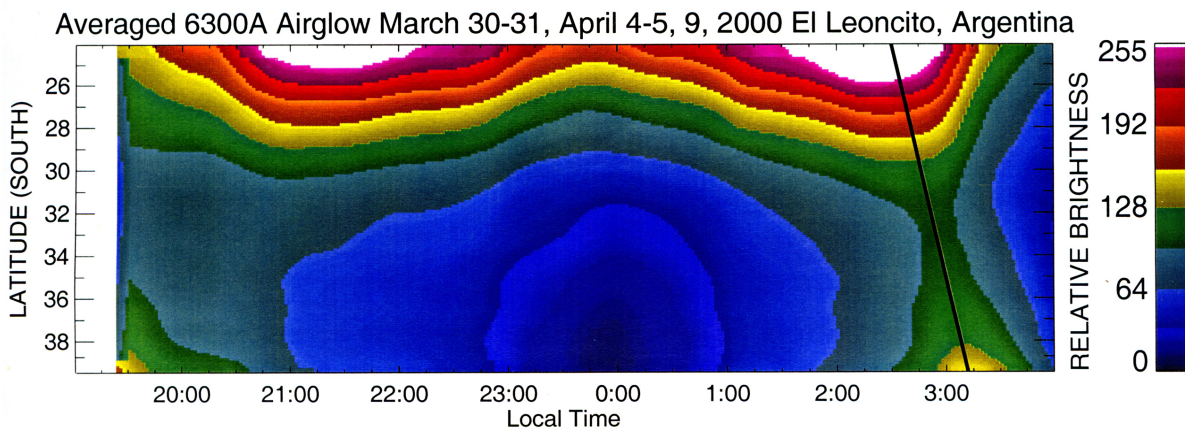

Fig. 5. Averaged meridional brightness vs. local time scan taken from $630.0 \mathrm{~nm}$ images on the nights of 4, 5 and 9 April, 2000. The brightness wave (BW) that originates from the midnight temperature maximum (MTM) near the equator is seen to reach and exit the El Leoncito FOV between 02:00 to 03:30 LT. The black line indicates the BW poleward propagation path, showing the highest geographic latitudes reached by a BW event (Colerico et al., 2006).
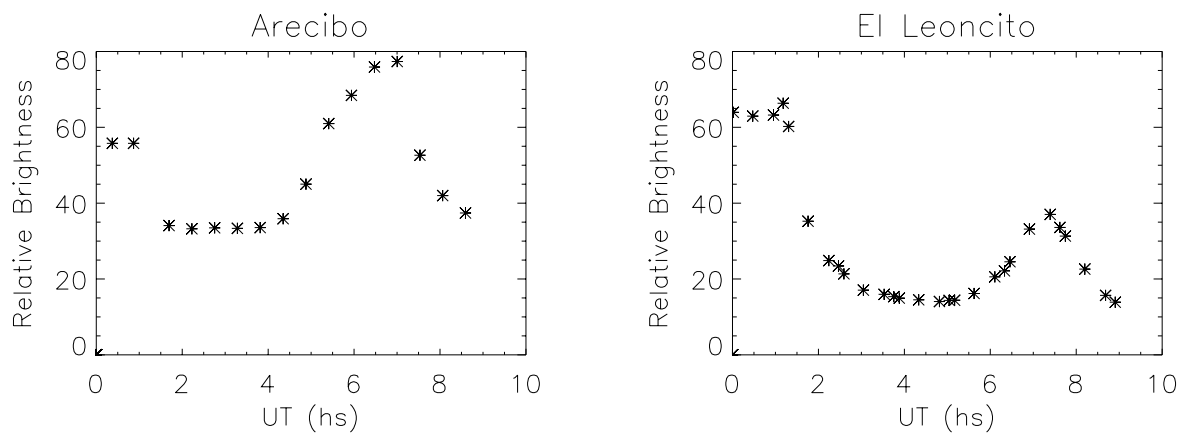

Fig. 6. Simultaneous detection of the passage of a BW at Arecibo and El Leoncito on 24 September 2003. Each point represents the average brightness of a 50 pixel-wide box centered at zenith. Notice the delay in the increase in brightness at the station further from the geographic equator (El Leoncito).

a brief time-history of the events on 29 September 2003 (top) and 26 February 2003 (bottom), showing the typically eastward drift of depletions in $630.0 \mathrm{~nm}$. These irregularities can also be detected when radio signal traverse the ionosphere. Figure 4 shows results from global positioning system (GPS) data measured at the nearby station UNSA $\left(24.73^{\circ} \mathrm{S}, 65.41^{\circ} \mathrm{W}\right)$ for the 26 February 2003 case, with unambiguous TEC depletions and phase fluctuations (dTEC/dt) clearly coincident with the optical features. Taken together, Figs. 2, 3 and 4 show El Leoncito to be an ideal location for multi-diagnostic studies of high-apex-height ESF phenomena in the South American longitude sector. Case studies of simultaneous detections of ESF-related airglow depletions at both Arecibo and El Leoncito are in progress.

\subsection{Brightness waves associated with the MTM}

A brightness wave (BW) observed by an all-sky imager is the optical signature of perturbation winds from the midnight temperature maximum (MTM), a large scale neutral temperature anomaly which originates in the thermosphere at low latitudes. The MTM is accompanied by a pressure increase causing a poleward reversal/abatement in the normal equatorwards meridional winds in the nighttime thermosphere. The downward displacement of ionospheric plasma by the modified winds produces an enhancement in the $630.0 \mathrm{~nm}$ airglow emission (Colerico et al., 1996; Colerico and Mendillo, 2002). While first detected at the Arequipa Observatory, its occurrence is not limited to low latitude regions. At Arecibo, it was seen to exit the northern edge of the FOV and therefore propagates to at least $24^{\circ} \mathrm{N}$ geographic latitude (Mendillo et al., 1997b). The benefit of setting up an imager at El Leoncito for BW research is that the poleward edge of its field of view occurs at $\sim 40^{\circ} \mathrm{S}$ geographic latitude.

As described by Colerico and Mendillo (2002), a convenient way to document $\mathrm{BW}$ events is to form a meridional brightness plot $(630.0 \mathrm{~nm}$ brightness on latitude vs. local time grid) by stacking N-S image scans for a full night. Colerico and Mendillo (2002) averaged fifteen nights of such scans to show unambiguously that BW events are a persistent, fixed in LT phenomena that appear in monthly mean patterns (recall that all-sky imaging systems operate only for a 2-week period each month). In Fig. 5, we show the average 


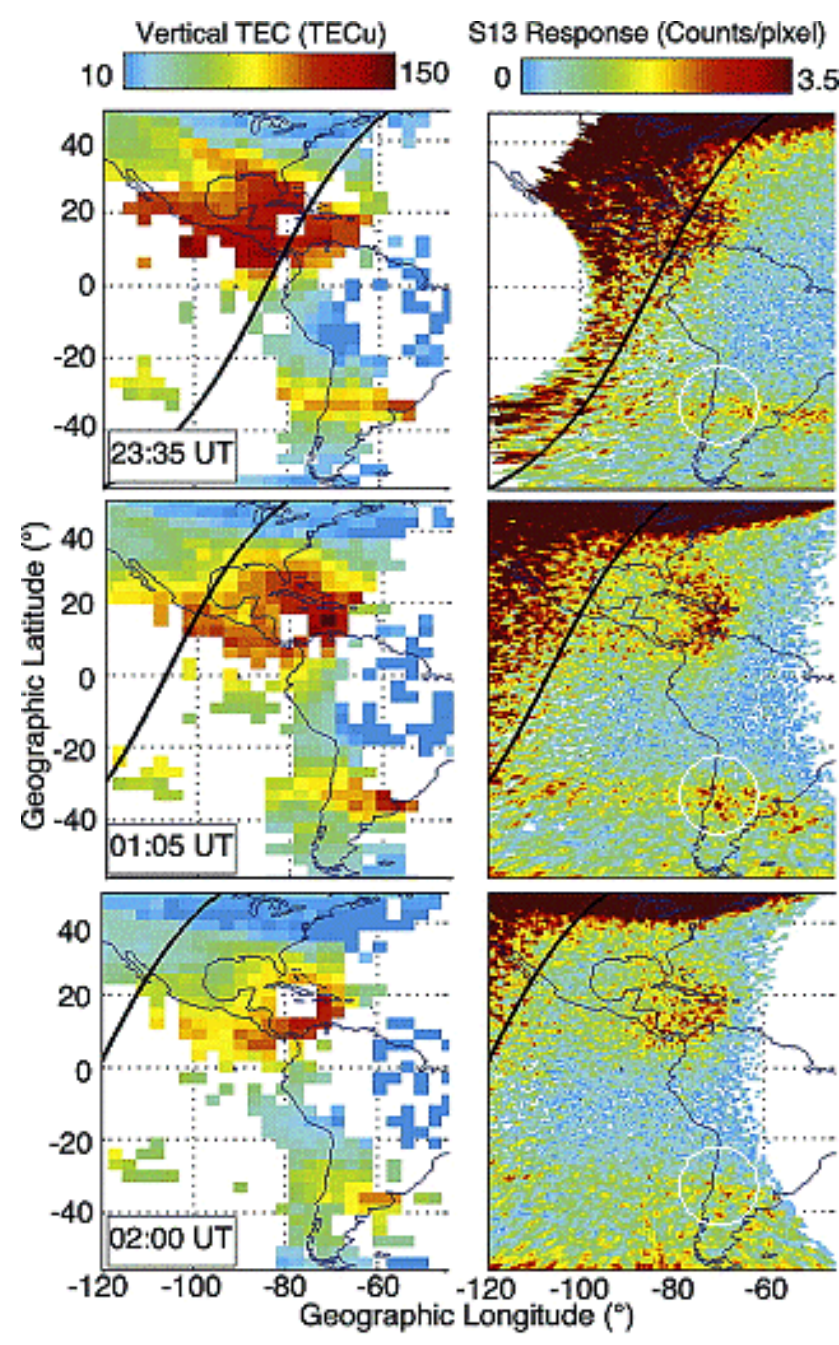

Fig. 7. GPS TEC (left panel) and IMAGE FUV (right panel) measurements during the large geomagnetic storm of 29-30 May 2003. White circles in the right panel represent the FOV of the all-sky camera at El Leoncito (adapted from Immel et al., 2005).

for three clear nights in March-April 2000 at El Leoncito, demonstrating the repeatable trends of these structures. This provides the first documentation of an unambiguous detection of a BW into midlatitudes. Case studies, showing the occurrence of BW at Arecibo and El Leoncito on the same nights, with El Leoncito BW lagging $\sim 1.5 \mathrm{~h}$ due to its more poleward $\mathrm{N}$ geographic latitude, have been found. Here we show a case on 24 September 2003 (Fig. 6). At each site, we averaged a 50 pixel-wide box centered at zenith and obtained an average relative brightness for the entire night. The arrival of the BW at Arecibo occurs at $\sim 04: 30$ UT, while at El Leoncito (located further away from the geographic equator) occurred later, at 06:00 UT. To drive such strong wind patterns, pronounced heating of the thermosphere at low latitudes is required. Yet, the temperature effect is not present in the empirical MSIS model (Hedin, 1983, 1991) of the neutral atmosphere, or in the horizontal wind model (HWM) of Hedin et al. (1996). To date, general circulation models have been unable to simulate successfully such MTM/BW effects (Colerico et al., 2006). Continuing observations of the BW events at El Leoncito will serve as a strong constraint on modeling MTM processes.

\subsection{Airglow enhancements associated with geomagnetic storms}

The response of the ionosphere's total electron content (TEC) at midlatitudes to geomagnetic activity, commonly called ionospheric storms, can be described as a short positive phase followed by a longer negative phase (Mendillo, 1971). During the magnetic storm of 29-30 May 2003, GPS networks and FUV data from the IMAGE satellite showed increases in both TEC and in the ultraviolet emissions from the Flayer during the period 20:00-02:00 UT in a localized region in South America (Immel et al., 2005). This storm produced an unusual long positive phase. During this storm the southward component of the solar wind , $B_{z}$, reached a peak negative value of $\sim-30 \mathrm{nT}$ at 19:00 UT and stayed south until $\sim$ 01:00 UT. The equatorial Dst reached a peak of $-130 \mathrm{nT}$ at 23:00 UT and a second minimum of $-131 \mathrm{nT}$ at 02:00 UT, while a maximum rate of change $\mathrm{dDst} / \mathrm{dt}$ of $\sim 23 \mathrm{nT} / \mathrm{h}$ occurred earlier at $\sim 22: 00$ UT. In Fig. 7 the GPS and IMAGE data show enhancements near $30^{\circ} \mathrm{S}$, the Southern Hemisphere counterpart of the F-layer enhancements seen in the Northern Hemisphere. The magnetic storm moves plasma not only to higher altitudes/latitudes, but also produces strong westward drifts for several hours, resulting in a co-rotating ionospheric plasma (Immel et al., 2005 and references therein). This is noticeable in the Northern Hemisphere where no significant plasma is observed to the East of the Caribbean.

For storm enhanced densities that linger past sunset, there should be pronounced increases in groundbased visible emissions (630.0 nm and $777.4 \mathrm{~nm}$ airglow emissions) due to the high recombination rate of a robust ionosphere (just as the normal inter-tropical arcs are the airglow signature of the TEC crests of the EIA). Airglow observations at El Leoncito detected unusual increases on this storm night and these are shown in Fig. 8. The top images show bright images in $777.4 \mathrm{~nm}$ between 00:22 and 01:56 UT spanning the entire FOV, coinciding with the appearance of the GPS TEC enhancements, and their IMAGE FUV signatures, into/out of the imager's FOV. The image at 02:56 UT shows "normal" brightness values. The dark feature to the right (east) is an airglow depletion that moves very slowly.

The images at the bottom in Fig. 8 show the corresponding $630.0 \mathrm{~nm}$ airglow images. Notice that the last image at 03:06 UT is the brightest. In order to follow the behavior during the entire night we averaged a 50 pixel-wide box centered at zenith. Figure 9 shows plots comparing the 


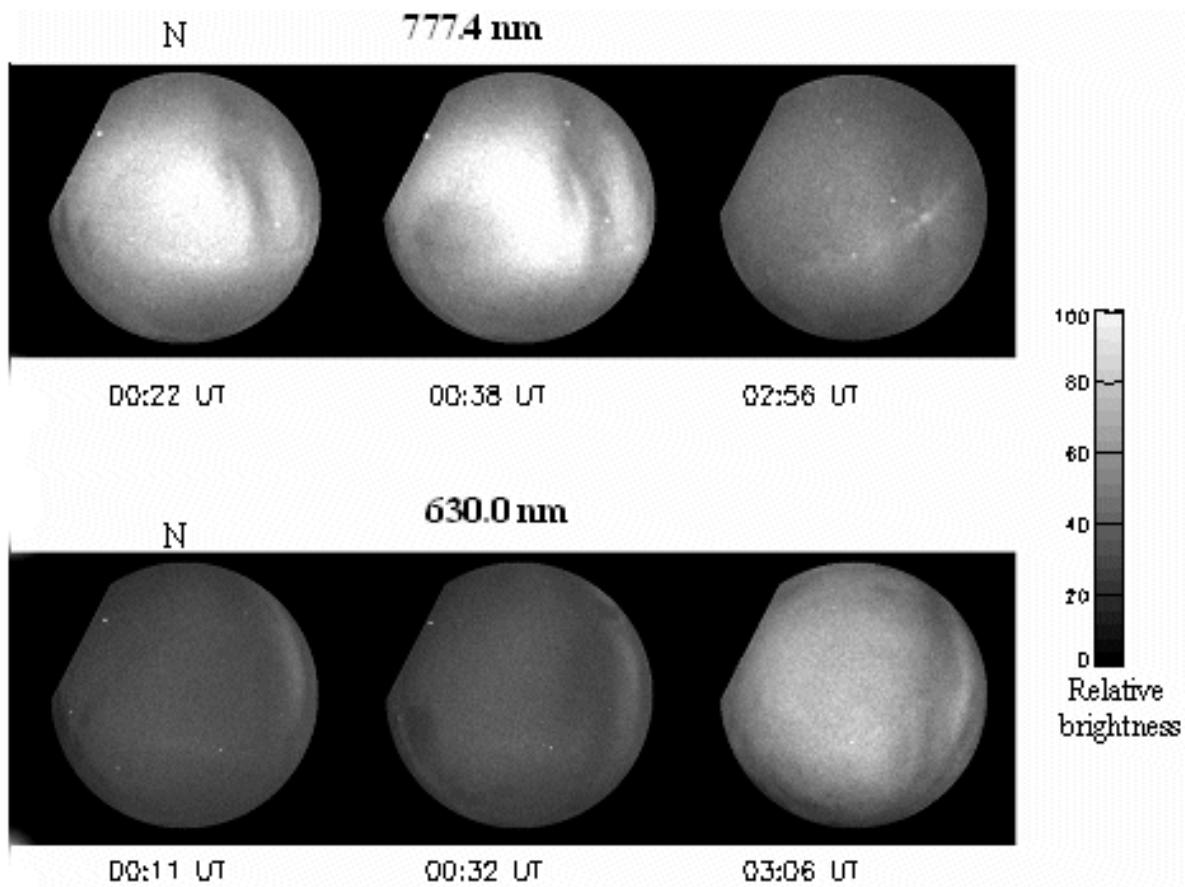

Fig. 8. (Top) High TEC values cause enhancements in $777.4 \mathrm{~nm}$ airglow images during the 29-30 May 2003 geomagnetic storm, in accordance with the results in Fig. 7; (bottom) $630.0 \mathrm{~nm}$ images.
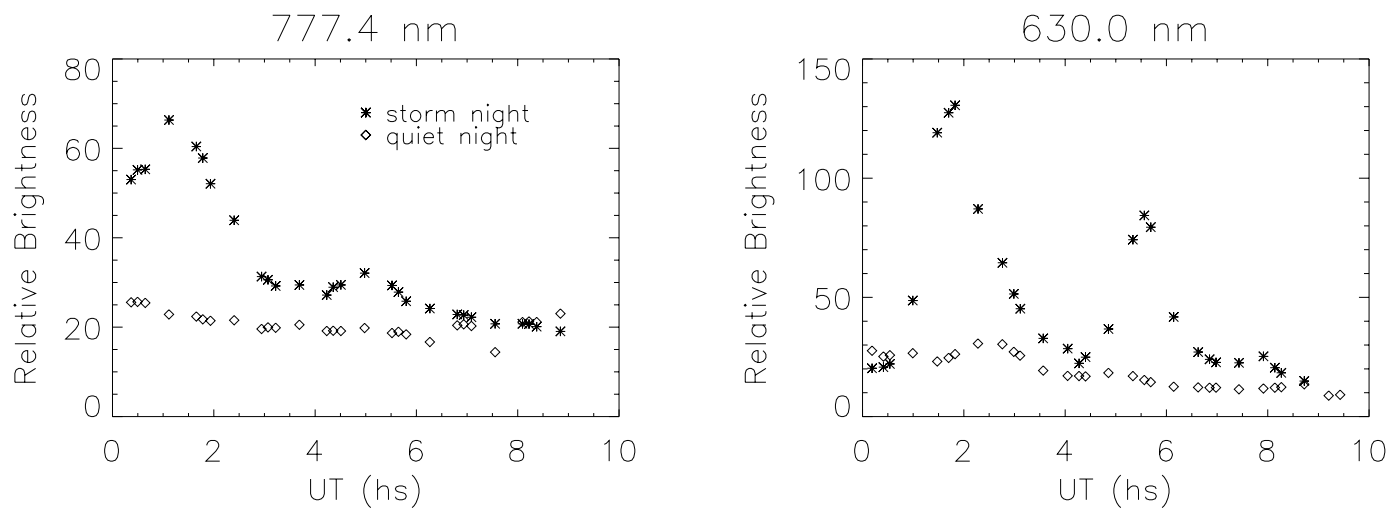

Fig. 9. Average brightness for $777.4 \mathrm{~nm}$ emission (left) and $630.0 \mathrm{~nm}$ (right). Stars indicate data points during the storm night; diamonds represent data for a "control night" (see text).

relative intensity of the 777.4 and $630.0 \mathrm{~nm}$ emissions for a "control night" (computed by taking the average of 15 clear nights without significant geomagnetic activity centered on 30 May) and the storm night. The error in each data point is $\sim 20-25 \%$. The large values for 777.4 from occur early in the night, while the brightness in the $630.0 \mathrm{~nm}$ peaks later, at $\sim 02: 00$ UT. It is interesting to note that the brightness in $630.0 \mathrm{~nm}$ shows two distinct peaks; the secondary peak seems to be also present in $777.4 \mathrm{~nm}$ at $\sim 05: 00 \mathrm{UT}$, but not as dramatic as the one in $630.0 \mathrm{~nm}$. Recall that $630.0 \mathrm{~nm}$ emissions depend, not only on the TEC, but also on the height of the F-layer. This result could indicate the presence of elec- tric fields that cause vertical motions that affect airglow production, perhaps associated with the two minima in Dst at 23:00 UT and 02:00 UT.

We examined other storm cases (6-7 April 2000 and 31 October 2003) and indeed found similar optical signatures: large $777.4 \mathrm{~nm}$ and $630.0 \mathrm{~nm}$ emissions. In all these cases, the universal time (UT) of maximum storm intensification (as characterized by dDst/dt or $B_{z}$ ) occurred at a local time near dusk at El Leoncito's longitude, the key requirement for growth of the electrodynamical "fountain effect" during storms (see Martinis et al., 2005, and references therein). 

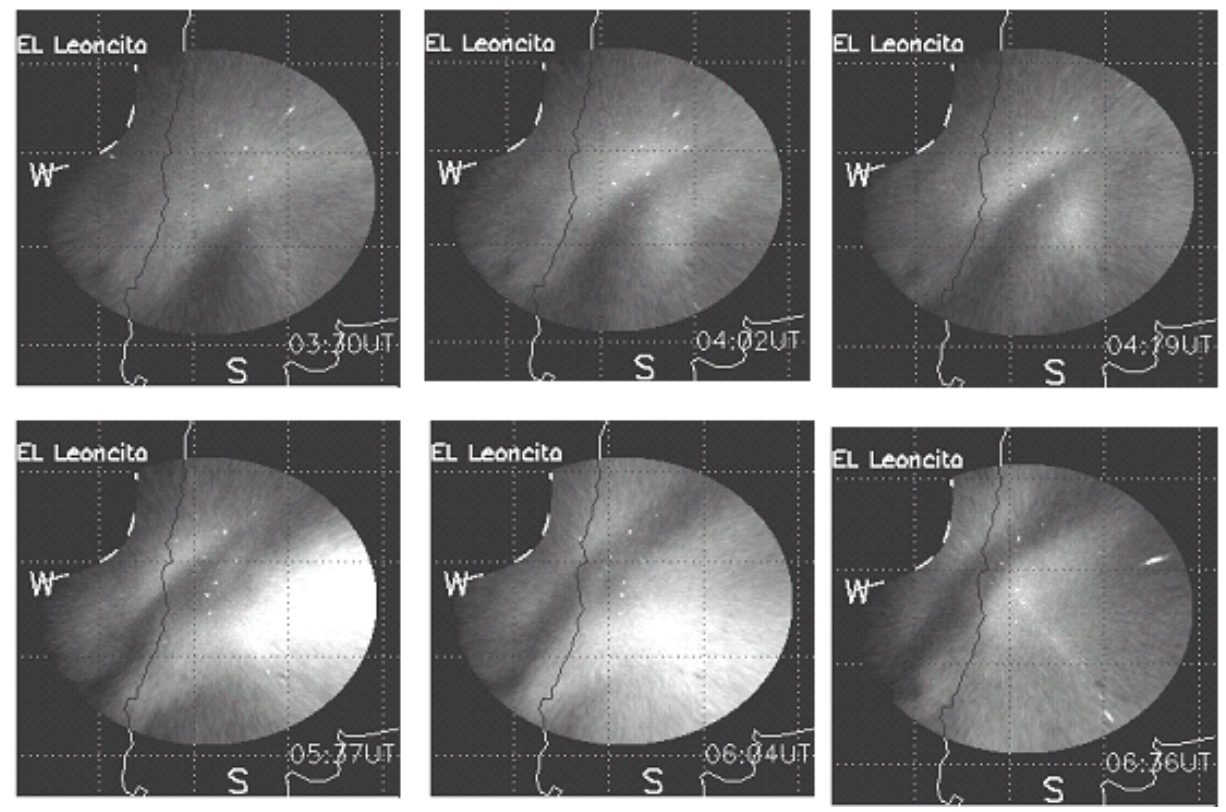

Fig. 10. Airglow depletions associated with mid-latitude instabilities detected by optical images in South America (19 December 2003). The $630.0 \mathrm{~nm}$ images are mapped to geographical coordinates ("unwarped" with $5^{\circ}$ latitude and longitude lines shown), with north at the top and west to the left. At 03:30 UT a dark band can be seen in the south-east of the FOV that subsequently moves north-westward (the direction predicted for a modified Perkins instability in the Southern Hemisphere).

\subsection{Airglow structures associated with mid-latitude insta- bilities}

Radars, satellites and imagers have been used to observe ionospheric mid-latitude instabilities (MLI), also known as mesoscale traveling ionospheric disturbances (MSTIDs), that are attributed to a variant of the Perkins Instability (Kelley and Makela, 2001). An important characteristic of these instabilities is that they tend to occur poleward of the crests of the equatorial ionization anomaly (EIA), and thus their investigation using an ISR and ground based imagers has been a central component of research at Arecibo. The radar signatures are strong reversals in perturbation electric fields, moving F-layer plasma upwards and downwards into regions of less or increased chemical loss, thus creating "bands" in the ionosphere (Benkhe, 1979). Optically, these features are readily observed in $630.0 \mathrm{~nm}$ data from Arecibo (Mendillo et al., 1997b; Garcia et al., 2000) and recently in dualhemisphere sites in the Asian longitude sector (Shiokawa et al., 2005). At Arecibo, they can occur as a single moving dark band in a bright background or as multiple dark bands moving towards the southwest (Garcia et al., 2000); thus, they have morphology characteristics quite distinct from the airglow depletions associated with ESF (i.e., highly structured and moving to the east, as in Figs. 2 and 3). Figure 10 shows an unambiguous detection of MLI bands in $630.0 \mathrm{~nm}$ airglow that occurred above El Leoncito on 19 December 2003. The original images were projected into geographic coordinates assuming an airglow height of $300 \mathrm{~km}$. The single-band structure with its movement towards the northwest in the Southern Hemisphere (as shown in Otsuka et al., 2004 and Shiokawa et al., 2005) make it distinct from the ESF and BW patterns seen frequently on other nights.

The only global study of MLI occurrence patterns was conducted by Saito et al. (1995) using the electric field and plasma density instruments on the Dynamics Explorer (DE) spacecraft. In the American longitude sector, they showed that Arecibo's location was close to the peak occurrence zone in their statistical summary, while that zone in the Southern Hemisphere was somewhat poleward of El Leoncito's location. While the DE dataset was limited in seasonal and solar cycle coverage, it is certainly consistent with our preliminary finding of limited detections of the MLI airglow signatures at El Leoncito.

Garcia et al. (2000) showed that, using linear theory, the maximum growth rate for the Perkins Instability can be written as:

$$
\begin{aligned}
\gamma= & \left(1 / 2 H_{n}\right)\left[\left(E_{o} \cos I\right) / B+U_{e} \sin \delta \cos I \sin I+\right. \\
& \left.\left(U_{e} \cos \delta+U_{s} \sin \delta\right) \cos I+U_{s} \cos \delta \cos I \sin I\right]
\end{aligned}
$$

where $H_{n}=$ neutral scale height, $E_{o}=$ radial electric field, $U_{e}=$ eastward wind, $U_{s}=$ southward wind, $\delta=$ magnetic declination, and $I=$ magnetic inclination.

Included in this expression are the effects due to neutral winds and ambient electric fields. For Arecibo, considered a site where typical features include the presence of 

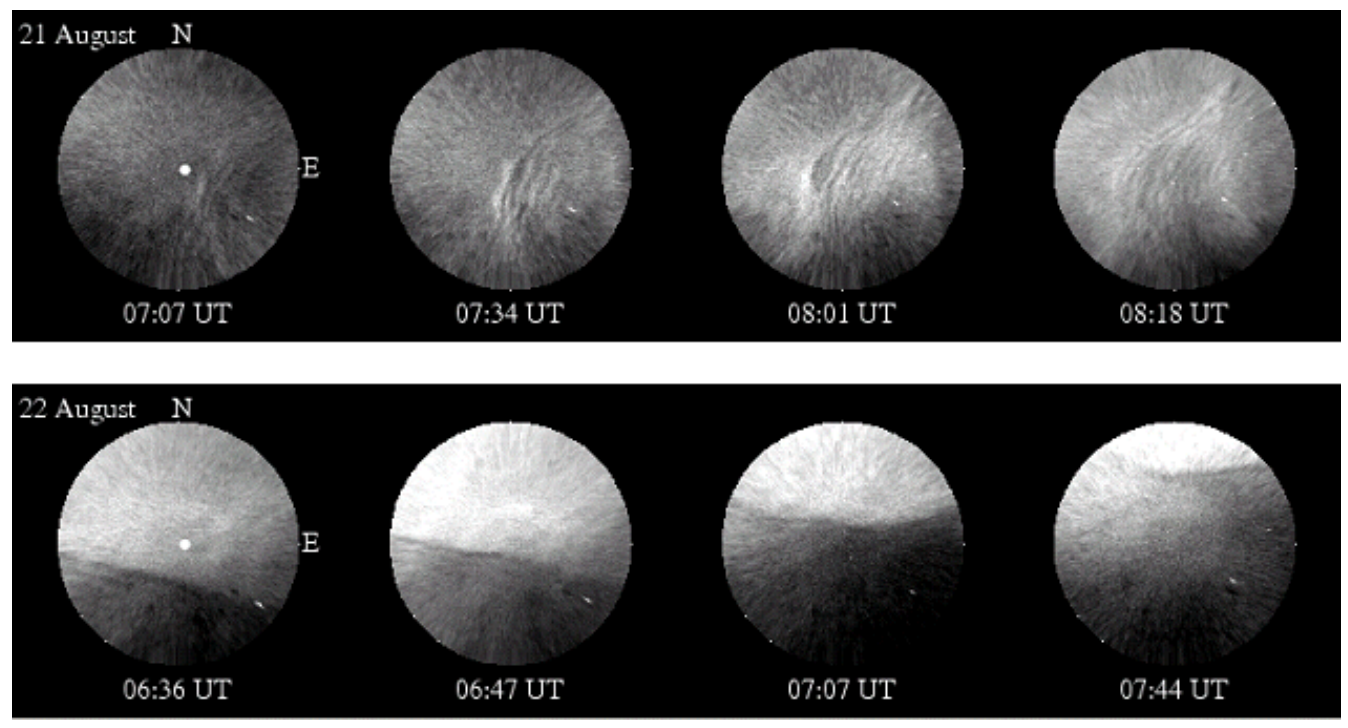

Fig. 11. Examples of mesospheric structures seen in geometrically-corrected $557.7 \mathrm{~nm}$ images. The top panel shows small scale gravity wave disturbances on 21 August 2001, exhibiting markedly curved wave-fronts indicative of a nearby source. The bottom panel shows a large scale mesospheric bore event propagating from the south on the subsequent night.

MLIs, a maximum value of $\sim 8 \times 10^{-4} \mathrm{~s}^{-1}$ was obtained in the calculations by Garcia et al. (2000). The geometric factors for Arecibo and El Leoncito are similar: for Arecibo $\delta=-10^{\circ}, I=45^{\circ}$; while for El Leoncito: $\delta=2.1^{\circ} ; I=34^{\circ}$. For El Leoncito, neutral winds, scale height, and magnetic field intensity were computed using TIEGCM, MSIS-90, and IGRF models, respectively for typical equinox conditions. For the radial electric field we assumed a threshold value of $1 \mathrm{mV} / \mathrm{m}$ (few $\mathrm{mV} / \mathrm{m}$ have frequently associated with this kind of instability, see Saito et al., 1995). We obtain a value for the growth rate of $1 \times 10^{-3} \mathrm{~s}^{-1}$, comparable to the maximum growth rate calculated for Arecibo. Thus, under these assumptions, MLI instabilities can occur at El Leoncito. A study of additional MLI detections with comparisons to the Saito et al. (1995) statistics is in preparation (Martinis et al., $\left.2006^{1}\right)$.

\section{Mesospheric structures}

4.1 Airglow structures associated with mesospheric dynamics

Nighttime airglow emissions at $557.7 \mathrm{~nm}$ can be used as a passive tracer of atmospheric dynamics in the mesosphere. Given the column-integrated nature of an airglow observation, to record structures in mesospheric green line the F-layer contribution to $557.7 \mathrm{~nm}$ must be small (usually

\footnotetext{
${ }^{1}$ Martinis, C., Mendillo, M., and Baumgardner, J. : Airglow observations of mid-latitude instabilities in the Southern Hemisphere, Geophys. Res. Lett., in preparation, 2006.
}

$<20 \%$ ). This is not always the case, and thus if strong F-layer structures exist (such as deep airglow depletions in $630.0 \mathrm{~nm}$ ) they are often present in $557.7 \mathrm{~nm}$ as well, and thus no mesospheric signatures can be studied during that portion of the night. More often than not, however, the mesospheric signal at $557.7 \mathrm{~nm}$ dominates image sets at that wavelength. Yet, due to the ICCD detector in use during this 5-year reporting period, it was challenging to find gravity wave events at their nominal brightness variations, e.g., the typically 1$5 \%$ brightness variations recorded at Millstone Hill where we employ a more sensitive bare-CCD detector (Smith et al., 2000). Nevertheless, bright events do occur, as shown in Fig. 11. Two large wave disturbances were recorded on two consecutive nights at El Leoncito in August 2001 (Smith et al., 2006). An example from 21 August 2001 is given in the top panel of Fig. 11. The brightness amplitudes of these small scale gravity waves were very much larger than typically observed (37\% in $\mathrm{O}\left({ }^{1} \mathrm{~S}\right)$ peak-to-peak). The highly curved nature of the wave fronts indicates that the source region was relatively localized and nearby, but a search of limited meteorological data on that night was not conclusive. Far more dramatic was the passage of the mesospheric bore event on 22 August 2001 (lower panel) that resulted in a strong reduction in the $\mathrm{O}\left({ }^{1} \mathrm{~S}\right)$ emission brightness due to an upward displacement in the $\mathrm{O}\left({ }^{1} \mathrm{~S}\right)$ layer height (Taylor et al., 1995; Smith et al., 2003). More thorough studies of mesospheric dynamics will be possible with a new detector installed at El Leoncito in early 2006, together with additional diagnostics systems from collaborating scientists. 


\section{Summary}

The Boston University airglow imager at the Complejo Astronómico El Leoncito (CASLEO) in Argentina has been in operation since mid-1999. Multi-spectral all-sky images of thermospheric and mesospheric emissions have been used to study a host of dynamical processes in the upper atmosphere. Using $630.0 \mathrm{~nm}$ observations, this location is well suited for investigations of depleted fluxtubes resulting from the Rayleigh-Taylor instability process that reach extraordinary apex heights $(>1800 \mathrm{~km}$ above the geomagnetic equator), as well as the influence of geomagnetic activity upon airglow depletions (Sahai et al., 1998). In addition, with its relatively high geographic latitude for a site at the equatorial ionization anomaly, the poleward extent of the $630.0 \mathrm{~nm}$ brightness wave due to the nighttime equatorial pressure bulge can be mapped well into mid-latitudes. The first case of the passage of a BW event in both hemispheres is reported. The time of occurrence is in agreement with a midnight temperature maximum occurring at the geographic equator.

A plasma instability typical of mid-latitudes has also been observed in the South American sector for the first time using ground-based optical systems. The northwestward motion is in agreement with optical observations in the Australian sector (Otsuka et al., 2004; Shiokawa et al., 2005). Its characteristics are consistent with a modified version of the Perkins instability, and thus quite distinct from the far more routine observations of ESF airglow depletions.

Episodic geomagnetic storms produce enhanced ionospheric TEC which has a strong airglow signature in the postsunset sector. Images at $777.4 \mathrm{~nm}$ show similar behavior to the spaced-based $1304 \mathrm{~nm}$ emissions from IMAGE, indicating an increase in the total electron content associated with the geomagnetic storm. Images at $630.0 \mathrm{~nm}$ show a more complicated pattern due to their dependence on vertical motions of the F-layer.

Finally, structures in $557.7 \mathrm{~nm}$ images provide opportunities to investigate the characteristics of mesospheric wave disturbances from a variety of atmospheric and orographic sources.

Based on this rich set of initial results, a new bare-CCD imaging system was installed in April 2006 to enable improved sensitivity during the solar minimum years.

Acknowledgements. We are grateful to H. Levato, Director of the Complejo Astronómico El Leoncito (CASLEO), and staff members for their strong support and valuable technical assistance throughout the course of this research. The all-sky camera instrumentation and its recent upgrade were funded by the Defense University Research Instrumentation Program (DURIP) via the Office of Naval Research (ONR). C. Martinis acknowledges the support from a CONICET Fellowship. Support for operations and scientific analysis come from ONR, with some student support from NSF. GPS data were obtained from the International GNSS Service network. We acknowledge the ACE Science Data Center for providing level 2 solar wind data, and the World Data Center for Geomagnetism, Kyoto for providing Dst data.

Topical Editor M. Pinnock thanks J. J. Makela and Y. Sahai for their help in evaluating this paper.

\section{References}

Aarons, J.: The longitudinal morphology of equatorial F-layer irregularities relevant to their occurrence, Space Sci. Rev., 63, 209-243, 1993.

Baumgardner, J., Flynn, B. and Mendillo, M. : Monochromatic imaging instrumentation for applications in aeronomy of the Earth and planets, Optical Eng., 32, 3028-3032, 1993.

Benkhe, R.: F Layer height bands in the nocturnal ionosphere over Arecibo, J. Geophys. Res., 84, A3, 974-978. 1979.

Colerico, M., Mendillo, M., Nottingham, D., Baumgardner, J., Meriwether, J., Mirick, J., Reinisch, B. W., Scali, J. L., Fesen, C., and Biondi, M.: Coordinated measurements of $F$ region dynamics related to the thermospheric midnight temperature maximum, J. Geophys. Res., 101, 26783, doi:10.1029/96JA02337, 1996.

Colerico, M. and Mendillo, M.: The current state of investigations regarding the thermospheric midnight temperature maximum (MTM), J. Atmos. Terr. Phys., 64, 1361-1369, 2002.

Colerico, M., Mendillo, M., Fesen, C., and Meriwether, J.: Comparative investigations of equatorial electrodynamics and low-tomid-latitude coupling of the thermosphere-ionosphere system in the American sector, Ann. Geophys. 24, 503-513, 2006.

Garcia, F., Kelley, M., Makela, J., and Huang, C.: Airglow obervations of mesoscale low-velocity traveling ionospheric disturbances at midlatitudes, 105, A8, J. Geophys. Res., $18407-$ $18415,2000$.

Hedin, A.: A Revised Thermospheric Model Based on Mass Spectrometer and Incoherent Scatter Data: MSIS-83, J. Geophys. Res., 88, 10 170-10 188, 1983.

Hedin, A.: Extension of the MSIS Thermospheric Model into the Middle and Lower Atmosphere, J. Geophys. Res., 96, 1159$1172,1991$.

Hedin, A., Fleming, E. L., Manson, A. H., et al.: Empirical wind model for the upper, middle and lower atmosphere, J. Atmos. Terr. Phys., 58, 1421-1447, 1996.

Immel, T. J., Foster, J., Coster, A., Mende, S., and Frey, H.: Global storm time plasma redistribution imaged from the ground and space, Geophys. Res. Lett., 32, doi:10.1029/2004GL021120, 2005.

Kelley, M., Garcia, F., Makela, J., Fan, T., Mak, E., Sia, C., and Alcocer, D.: Highly structured tropical airglow and TEC signatures during strong geomagnetic activity, Geophys. Res. Lett., 27, 465, doi:10.1029/1999GL900598, 2000.

Kelley, M. and Makela, J.: Resolution of the discrepancy between experiment and theory of midlatitude F-region structures, Geophys. Res. Lett., 28, 2589-2592, 2001.

Kelley, M., Makela, J., and Saito A.: The mid-latitude F region at the mesoscale: some progress at last, J. Atm. Sol. Terr. Phys., 64, 1525-1529, 2002.

Martinis C., Eccles, J. V., Baumgardner, J., Manzano, R. ., and Mendillo, M.: Latitude dependence of zonal plasma drifts obtained from dual-site airglow observations, J. Geophys. Res., 108 (A3), 1129, doi:10.1029/2002JA009462, 2003. 
Martinis, C., Mendillo, M. J., and Aarons, J.: Toward a synthesis of equatorial spread $\mathrm{F}$ onset and suppression during geomagnetic storms, J. Geophys. Res., 110, doi:10.1029/2003JA010362, 2005.

Mendillo, M.: Ionospheric Total Electron Content Behavior During Geomagnetic Storms, Nature, 234, 23-24, 1971.

Mendillo, M. and Baumgardner, J.: Airglow Characteristics of Equatorial Plasma Depletions, J. Geophys. Res., 87, 7641-7652, 1982.

Mendillo, M., Spence, H., and Zalesack, S.: Simulation Studies of Ionospheric Airglow Signatures of Plasma Depletions at the Equator., J. Atmos. Terr. Phys., 47, 885-891, 1985.

Mendillo, M., Baumgardner, J., Pi, X., Sultan, P., and Tsunoda, R.: Onset Conditions for Equatorial Spread-F, J. Geophys. Res., 9, 13 865-13 876, 1992.

Mendillo, M., Baumgardner, J., Colerico, M., and Nottingham, D.: Imaging science contributions to equatorial aeronomy: Initial results from the MISETA program, J. Atmos. Sol. Terr. Phys., 59, 1587-1599, 1997a.

Mendillo, M., Baumgardner, J., Nottingham, D., Aarons, J., Reinisch, B., Scali, J., and Kelley, M.: Investigations of thermospheric-ionospheric dynamics with $6300-\AA$ images from the Arecibo Observatory, J. Geophys. Res., 102, 7331-7344, doi: 10.1029/96JA02786, 1997b.

Mendillo M., Zesta, E., Shodhan, S., et al.: Observations and Modeling of the Coupled Latitude-Altitude Patterns of Equatorial Plasma Depletions, J. Geophys. Res., doi:10.1029/2005JA011157, 2005.

Miller, C. A., Swartz, W. E., Kelley, M. C., Mendillo, M., Nottingham, D., Scali, J., and Reinisch, B.: Electrodynamics of midlatitude spread F 1. Observations of unstable, gravity wave-induced ionospheric electric fields at tropical latitudes, J. Geophys. Res., 102, 11521, doi:10.1029/96JA03839, 1997.

Otsuka, Y., Shiokawa, K., Ogawa, T., and Wilkinson, P.: Geomagnetic conjugate observations of medium-scale traveling ionospheric disturbances at midlatitude using allsky airglow imagers, Geophys. Res. Lett., 31, L15803, doi:10.1029/2004GL020262, 2004.
Perkins, F.: Spread F and ionospheric currents, J. Geophys. Res., 78, 218-226, 1973.

Sahai, Y., Fagundes, P. R., Bittencourt, J., and Abdu, M.: Occurrence of large scale equatorial F-region plasma depletions during geomagnetic disturbances, J. Atmos. Sol. Terr. Phys., 60, 15931604, 1998.

Sahai, Y., Fagundes, P. R., and Bittencourt, J. A.: Transequatorial F-region ionospheric plasma bubbles: solar cycle effects, J. Atm. Sol. Terr. Phys., 62, 1377-1383, 2000.

Saito, A., Iyemori, T., Sugiura, M., Maynard, N. C., Aggson, T. L., Brace, L. H., Takeda, M., and Yamamoto, M.: Conjugate occurrence of the electric field fluctuations in the nighttime Midlatitude ionosphere, J. Geophys. Res., 100, 21439, doi:10.1029/95JA01505, 1995.

Shiokawa, K., Otsuka, Y., Tsugawa, T., et al.: Geomagnetic conjugate observation of nighttime medium-scale and large-scale traveling ionospheric disturbances: FROMT3 campaign, J. Geophys. Res. 110, A05303, doi:10.1029/2004JA010845, 2005.

Smith, S., Mendillo, M., Baumgardner, J., and Clarke, R.: Mesospheric Gravity Wave imaging from a sub-auroral site: First results from Millstone Hill, J. Geophys. Res., 105, 27 119-27 130, 2000.

Smith, S. M., Taylor, M. J., Swenson, G. R., She, C. Y., Hocking, W., Baumgardner, J., and Mendillo, M.: A Multi-Diagnostic Investigation of the Mesospheric Bore Phenomenon, J. Geophys. Res., 108, 13-30, 2003.

Sobral, J., Abdu, M., Takahashi, H., et al.: Ionospheric plasma bubble climatology over Brazil based on 22 years (1977-1998) of $630 \mathrm{~nm}$ airglow observations, J. Atmos. Sol. Terr. Phys., 64, 1517-1524, 2002.

Taylor, M. J., Turnbill, D. N., and Lowe, R. P.: Spectrometric and imaging measurements of a spectacular gravity wave event observed during the ALOHA-93 campaign, Geophys. Res. Lett., 22, 2849-2852, 1995.

Tsunoda, R.: Control of the Seasonal and Longitudinal Occurrence of Equatorial Scintillations by the Longitudinal Gradient in Integrated E Region Pedersen Conductivity, J. Geophys. Res., 90, 447-456, 1985. 